\title{
UNA CONCEPCIÓN SEMÁNTICA RETICULAR DE LOS VALORES
}

\author{
Luis Miguel Peris-Viñé*
}

\begin{abstract}
RESUMEN
El trasfondo de este trabajo es la conexión entre conocimiento y acción a través de los valores. Los valores contribuyen a diseñar ciertas líneas programáticas de esa conexión. En este trabajo defenderé una concepción general sobre los valores que permite comprender las relaciones entre hechos y valores y superar las restricciones de la dicotomía hecho-valor. Se parte de la distinción entre la identificación de un valor y la evaluación del mismo. Los valores son concebidos como realizaciones de los hechos que pueden ser reconstruidos como propiedades teóricas de los mismos usando los recursos cognitivos con los que manejamos usualmente las propiedades teóricas que asignamos al mundo. Se trata de una concepción semántica de los valores en la que se asume una configuración reticular de las interrelaciones entre valores y sus realizaciones. Creemos que dicha concepción, aunque posee sustentos propios, encuentra apoyos en algunos desarrollos de la filosofía de la ciencia contemporánea. En particular, para defender esta idea, mostraremos cómo la metateoría estructural, el principal desarrollo de los enfoques semánticos de la actual filosofía de la ciencia, posee recursos para expresar ciertos aspectos de la esa concepción sobre los valores. Este resultado de nuestra discusión tiene una consecuencia de largo alcance, pues apoyaría la idea de que la filosofía de la ciencia es una materia relevante para la configuración de la axiología.

PALABRAS CLAVE: valores - ideología - hechos - metaética - metateoría estructural
\end{abstract}

\section{RESUMO}

O pano de fundo deste trabalho é a conexão entre o conhecimento e a ação através de valores. Os valores contribuem para o desenho de certas linhas programáticas dessa conexão. Neste artigo defenderei uma concepção geral de valores que permita compreender as relações entre fatos e valores e ultrapassar os constrangimentos da dicotomia fatos-valor. O ponto de partida é a distinção entre a identificação de um valor e a sua avaliação. Os valores são concebidos como realizações de fatos que podem ser reconstruídos como propriedades teóricas dos mesmos valores usando os recursos cognitivos com os quais normalmente lidamos com as propriedades teóricas que atribuímos ao mundo. É uma concepção semântica de valor em que se assume uma configuração reticular das inter-relações entre valores e suas realizações. Acreditamos que essa concepção, embora tenha seus próprios suportes, encontra apoio em alguns desenvolvimentos da filosofia da ciência contemporânea. Em particular, para defender esta ideia, mostraremos como a metateoria estrutural, principal desenvolvimento das abordagens semânticas da filosofia da ciência atual, possui recursos para expressar certos aspectos dessa concepção sobre valor. Este resultado da nossa discussão tem uma consequência de grande alcance, uma vez que apoiaria a ideia de que a filosofia da ciência é um assunto relevante para a configuração da axiologia.

PALAVRAS-CHAVE: valores - ideologia - fatos - metaética - metateoria estrutural

\footnotetext{
*Universidad de Granada. perisv@ugr.es.

Perspectivas - Revista do Programa de Pós-Graduação em Filosofia da UFT - n. 2 - 2018
} 


\section{Introducción: configuración axiológica del conocimiento y representación cognitiva de valores}

Cuando se plantean las relaciones entre conocimiento y valores podemos distinguir dos trayectos de posibles relaciones: el que va de los valores al conocimiento y el que va del conocimiento a los valores. La comprensión plena de las conexiones entre conocimiento y valores requiere comprender cómo transitamos por ambos trayectos. En el primer trayecto podemos abordar la configuración del conocimiento mediante los valores, mientras que en el segundo trayecto podemos abordar la representación de los valores mediante el conocimiento. Es decir, al recorrer el primer trayecto nos ocupamos de qué valores están presentes en las distintas formas de conocimiento y en particular en la ciencia y cómo influyen en su desarrollo, mientras que al recorrer el segundo trayecto nos ocupamos de cómo describir o identificar valores mediante los instrumentos del conocimiento.

En el desarrollo de cada uno de estos dos trayectos es posible plantear diversas cuestiones relativas al papel del conocimiento. Esas cuestiones muestran la existencia de una tensión entre el conocimiento y los valores, un desajuste entre ambos. Tales cuestiones se plantean respecto de si el conocimiento está o debe estar libre de valores y respecto de si el conocimiento nos sirve o no para representar valores. Estas tensiones se han manifestado respectivamente en los debates sobre la posibilidad de una ciencia libre de valores y sobre la posibilidad del cognitivismo axiológico. En ambos territorios se han defendido versiones diversas de lo que cabe entender como escisión entre conocimiento y valores. Por tanto, por 'escisión entre conocimiento y valores' cabe entender dos cosas, según en qué trayecto lo estemos planteando: los valores no configuran el conocimiento, el conocimiento no sirve para representar valores.

Obviamente el estudio de ambos trayectos no es independiente. La defensa de un papel para los valores en la configuración del conocimiento necesita apostar por alguna solución al problema de la representación de los valores mediante el conocimiento, pues comprender el papel de los valores requiere que previamente sepamos cómo representarlos. Si no solucionamos el problema de la representación de los valores no solucionaremos el problema de la contribución de los valores al conocimiento. Esto lo que indica es una prioridad de la cuestión de la representación de valores frente a la cuestión de la configuración del conocimiento mediante valores. 
En el primer trayecto, el de la configuración axiológica del conocimiento, se discute si los valores pueden guiar la configuración del conocimiento. Por ello, un requisito para comprender esa posible configuración es captar la conexión que existe entre el ámbito de la teoría y el ámbito de la práctica. En el segundo trayecto, el de la representación cognitiva de los valores, se discute si el conocimiento permite representar los valores. Un requisito para abordar esta posibilidad es disponer de una concepción general sobre los valores. El principal objetivo del presente escrito es responder a ese requisito y ofrecer una concepción general sobre los valores. Parte central de esa concepción general sobre los valores aquí defendida es la tesis de que los valores son propiedades teóricas del mundo que se realizan en los hechos.

\section{Distintas acciones respecto de los valores}

Los valores están presentes por doquier, en todos los grandes territorios de la actividad humana. Los valores permiten la constitución del conocimiento, fundamentan la práctica y guían la acción. Pero encontramos múltiples dificultades para precisar el papel de los valores en tales procesos. Esas dificultades se deben, en parte, a la diversidad y complejidad de las acciones que efectuamos respecto de los valores y a las múltiples interrelaciones existentes entre esas acciones. El estudio de los valores debería iniciarse tomando conciencia que respecto de los valores podemos hacer distintas cosas, que podemos llevar a cabo distintas acciones respecto de los valores. Además, algunas de las dificultades que apreciamos en las discusiones sobre los valores proceden de no distinguir correctamente esas distintas acciones. La naturaleza, los requisitos y las implicaciones de tales acciones no son equivalentes. Su distinción y análisis es un requisito para abordar las cuestiones centrales sobre los valores.

Atendamos a las distintas acciones o tareas que, como agentes usuarios o como agentes teóricos, podemos desarrollar en nuestra interacción con los valores. Al hacerlo aparecerán las distintas cosas que podemos constituir respecto de los valores. Aunque no completa, la siguiente lista es representativa: conceptualizar un valor, describir los hechos o sistemas en los que puede realizarse un valor, identificar un valor en los hechos o sistemas donde se realiza, evaluar un valor, tener o disponer de un valor, recomendar un valor, desear un valor, imponer un valor, ocultar un valor, reprimir un valor. 
Entre esas acciones existen relaciones diversas. Algunas de estas acciones se realizan en determinado orden, otras son dependientes entre sí, y algunas de ellas están incluidas en otras. Aunque también algunas son independientes entre sí. Por ejemplo, necesitamos conceptualizar previamente un valor para identificarlo, y para posteriormente evaluarlo, elegirlo o para recomendarlo; aunque no parece necesario evaluar un valor para conceptualizarlo ni para identificarlo si parece necesario haberlo identificado para evaluarlo; en cambio podemos tener un valor sin haberlo identificado; también podemos recomendar o imponer un valor sin tenerlo o sin haberlo identificado previamente; etc.

\section{Evaluación e identificación de valores}

En la literatura especializada el tratamiento de los valores se ha realizado casi de modo exclusivo a través de su mención en juicios de valor, es decir se ha priorizado el análisis de la evaluación de los valores. Esto se aprecia en que los debates suelen atender a los factores y circunstancias que afectan a la elección o rechazo de valores por un agente. Sin embargo, como hemos señalado, respecto de los valores, bien como agentes usuarios o bien como agentes teóricos, podemos desarrollar diferentes acciones o tareas, distintas e independientes de su evaluación. En los juicios de valor un agente expresa la aceptación o el rechazo de cierto valor como resultado de una evaluación en la que quizás se ha comparado dicho valor con otros valores u otros aspectos relevantes, bien siguiendo opciones relativas u opciones puras. Es decir, los juicios de valor pueden tener un alcance relativo o un alcance absoluto. ${ }^{1}$

Para la elaboración de la concepción general sobre los valores aquí propuesta, se le dará prioridad no a la evaluación de valores sino a la identificación de valores. El darle prioridad a la identificación se justifica porque asumimos, como una posición intuitiva de partida, que es posible identificar valores sin necesidad de evaluar esos valores. Es decir, asumimos lo que podemos denominar el principio de la independencia evaluativa de la identificación de valores. Además de asumir esa independencia, más abajo, en la sección dedicada al papel de las ideologías, intentaremos mostrar cómo las ideologías pueden ser

\footnotetext{
${ }^{1}$ La primacía de la evaluación se aprecia en el compromiso clásico de que bueno y malo son los valores centrales de los que se ocupa la ética. (MOORE, 1903) constituye una fuente de propuestas y análisis contemporáneos de ese compromiso sobre el objeto de estudio de la ética. En diversos terrenos se ha seguido discutiendo y asumiendo la primacía de la evaluación en el tratamiento de los valores. En particular en los debates sobre la dicotomía hecho-valor (MACKIE, 1977 y PUTNAM, 2002) y en el análisis de los conceptos thin y los conceptos thick (HARE, 1952 y WILLIAMS, 1985).
}

Perspectivas - Revista do Programa de Pós-Graduação em Filosofia da UFT - n. 2 - 2018 
guías tanto para la identificación como para la evaluación, pero guías independientes. Lo cual debe ser entendido como razones para defender el principio de la independencia evaluativa de la identificación de valores.

De modo alternativo, quienes se opongan a ese principio, $\mathrm{y}$ en general quienes asuman la prioridad de la evaluación frente a la identificación de valores, deberán defender el principio de la dependencia evaluativa de la identificación, según el cual no es posible identificar valores sin evaluarlos. Aunque no se defienda explícitamente, este principio es asumido frecuentemente en las discusiones sobre los valores. Las razones para su asunción deberían encontrarse siguiendo algunas de las siguientes líneas argumentativas que son parcialmente coincidentes e interdependientes: (1) no podemos identificar valores sin evaluarlos. Las argumentaciones en esta línea señalan que no es posible establecer que algo tiene o realiza cierto valor sin formular un juicio evaluativo sobre ese algo. Habría dos estadios, el de identificar y el de evaluar, y el primero no puede completarse hasta no haberse completado el segundo; (2) existe un acto epistémico único consistente en identificar y evaluar a la vez. Según esta línea argumentativa no habría dos estadios, el de identificar y el de evaluar, sino uno sólo, el de identificar-evaluar; (3) todos los valores contienen esencialmente un componente, el bien/mal, cuya identificación consiste en evaluarlo. Según esta línea, si los valores son así, si los valores contienen ese componente, si no existen sin el bien o el mal que un agente les atribuye tras evaluarlos, entonces identificar un valor conlleva necesariamente un acto de evaluación. Una variante de esta posición consiste en asumir que bueno y malo, como tales, son valores morales.

Nuestra defensa del principio de la independencia evaluativa de la identificación de valores se basa en que, desde la práctica y desde la teoría, se puede distinguir el estadio de identificación del estadio de evaluación, por lo cual, entonces, los valores no estarán constituidos por el resultado de la evaluación (por ejemplo, por el bien o por el mal). Y a la postre lo que sea un valor no podrá depender de si alguien lo elige o lo rechaza tras una evaluación, o si expresa su elección mediante un juicio de valor. Los agentes usuarios pueden realizar la identificación y la evaluación como dos acciones distintas, tan distintas que a veces sólo efectúan la primera y no la segunda. Y los agentes teóricos pueden caracterizarlas de modo independiente. Estos serían casos de ausencia de evaluación. También los casos de evaluaciones divergentes apoyan este principio, casos en los que un mismo valor puede recibir evaluaciones divergentes o contrapuestas. Estos casos se Perspectivas - Revista do Programa de Pós-Graduação em Filosofia da UFT - n. 2 - 2018 
justifican si la identificación es previa y no presupone ni conlleva ningún acto de evaluación.

Desde nuestra perspectiva, bueno y malo no son valores, ni componentes que formen parte de los valores. Son conclusiones de un agente que califica de esa forma ciertos valores en atención a su conocimiento y a sus elecciones. Dichos calificativos forman parte de juicios morales, pero no de enunciados de identificación de los valores morales afectados. En general, no forma parte de lo que es ser un valor el ser bueno o ser malo para alguien o en absoluto. Los valores no son aquello que nos parece bueno ni el ser bueno que le adscribimos a algo, sino algo que identificamos en el mundo, que a veces aparece como una consecuencia de un hecho y que, quizás, puede parecernos bueno o malo, o quizás no nos parece ni bueno ni malo, porque no nos induce ningún juicio evaluativo. Lo que sea básicamente un valor vendrá establecido por los modos de identificar valores que los usuarios emplean y que los teóricos reconstruyen.

Los enunciados de identificación de valores establecen la presencia o ausencia de cierto valor en cierto hecho, objeto u acción. Dicha presencia puede concebirse como la realización de cierto valor conceptualizado previamente. Dado que un mismo valor puede realizarse en distintos hechos, puede hablarse de instancias distintas de un mismo valor. La instancia de un valor es el modo particular en el que ese valor se realiza en un determinado hecho.

No obstante, pese a que nuestra concepción general sobre los valores se basa en la identificación y no en la evaluación de valores, los procesos de evaluación deberían poder ser analizados una vez caracterizados los procesos de identificación de valores. Es decir, los juicios de valor y lo que se sigue de ellos, por ejemplo, la elección y la bondad de los valores, también deberían ser reconstruidos dentro de esta perspectiva que prioriza los procesos de identificación. Para ello habría que añadir a los enunciados de identificación correspondientes otros factores pertinentes ulteriores.

\section{Consecuencias de partir de los procesos de identificación}

Partir del principio de la independencia evaluativa de la identificación de valores abre las puertas al empleo de aquellos recursos cognitivos que usamos en ciertos contextos para establecer la presencia o ausencia de ciertas cosas en el mundo sin la necesidad de tener en cuenta si algún agente acepta o rechaza esas cosas. En esos contextos describimos hechos e

Perspectivas - Revista do Programa de Pós-Graduação em Filosofia da UFT - n. 2 - 2018 
identificamos alguna de sus propiedades sin requerir partir de la aceptación o rechazo de esa propiedad por parte de algún agente. Esta estrategia cognitiva la podríamos trasladar al campo de los valores si es que asumimos el principio de la independencia evaluativa de la identificación de valores. Aceptando esa estrategia, lo que defiendo en este punto respecto de los valores es que en los procesos de identificación de ciertas propiedades cognoscibles usuales y en los procesos de identificación de los valores se emplean recursos parcialmente semejantes, y que también son semejantes las dificultades que conllevan. Entre esos recursos cognitivos se encuentran no sólo la ciencia, - en sus distintas manifestaciones y bajo la forma de teorías-, sino también las metateorías y, en particular, la filosofía de la ciencia.

Por otro lado, este empleo de recursos cognitivos usuales para la identificación de valores sería una razón que apoya la concepción de los valores como propiedades asignables a los hechos (las cosas, los sucesos, los procesos, las acciones). Si los valores pueden ser identificados con los recursos con los que identificamos diferentes propiedades en el mundo entonces los valores son propiedades semejantes respecto de su identificación a las primeras. Aquí está operando una apuesta general para abordar la ontología a partir de la epistemología. En el caso de los valores ello supone decir qué tipo de cosas son los valores a partir de la comprensión de los procesos que llevan a su identificación. Los valores serían propiedades semejantes a otras que asignamos al mundo porque para su identificación empleamos recursos semejantes.

Otra de las consecuencias ventajosas del uso del principio de la independencia evaluativa es que permite demarcar con claridad el tipo de contribución de los sujetos en las distintas etapas de su relación con los valores, y en particular acotar con precisión la contribución de sus compromisos subjetivos.

\section{Hechos, valores y evaluación de valores}

Parto de una perspectiva básica según la cual un valor, en un sentido intuitivo y genérico, es un logro o resultado de los hechos y de los objetos. Más en concreto, los valores son consecuencias que se derivarían de la existencia de cierto objeto, de la ocurrencia de cierto suceso o de la realización de cierta acción, independientemente de si son consecuencias deseadas o no por un agente, y de si son consideradas buenas o malas por él. El agente 
puede adscribir un valor a un hecho en el sentido de afirmar que el valor está presente en ese hecho. Obviamente también puede negar su presencia en ese hecho.

Esta noción de valor diverge de aquella otra noción en la que un valor es una etiqueta con la que los agentes expresan su aceptación o rechazo de cierto contenido. El problema de esta segunda noción es que nos impide comprender aspectos básicos del modo en el que tratamos los valores, aspectos relacionados con la distinción entre el valor y la evaluación de ese valor. Me opongo a esta segunda noción porque un valor no es la etiqueta que un agente atribuye al evaluar o calificar algo mediante un juicio, sino un algo que un agente puede o no evaluar y calificar. El calificativo suele implicar o equivaler a la aceptación o el rechazo de ese valor. Pero el valor no es el calificativo fruto de la evaluación, sino el algo evaluado o calificado. El valor es el contenido calificado por la etiqueta evaluativa, no la etiqueta evaluativa. El fruto de la evaluación suele expresarse atribuyendo bondad o maldad a algo. Pero los valores no están constituidos por el bien o por el mal atribuido, sino por aquello a lo que se lo atribuimos.

Por tanto, la identidad de los valores no está determinada por los juicios de valor de los agentes, que son juicios en los que normalmente atribuimos bondad o maldad a algo, sino por los procesos de identificación, y los enunciados correspondientes, de ese algo que enjuiciamos como bueno o malo. Por otro lado, ese algo calificado no es un hecho, sino un atributo que asignamos a un hecho. Un valor es un atributo que los agentes pueden adscribir a los hechos. Los agentes pueden o no calificar ese atributo, y ese calificativo puede a veces implicar la aceptación o el rechazo del atributo por parte del agente. Estos componentes pertenecen a niveles distintos y en ellos se opera de manera sucesiva. La secuencia es la siguiente:

$\langle$ hecho, atributo o valor, calificativo del valor + aceptación o rechazo $\rangle$

Pese a las diferencias entre hechos, valores y evaluación de valores, y a la existencia de dos momentos, el momento de la identificación y el momento de la evaluación, en ocasiones no somos conscientes de la existencia de los mismos. Estos dos momentos pueden darse conjuntamente. Como en el siguiente ejemplo:

eludir impuestos es insolidario y eso está mal

Perspectivas - Revista do Programa de Pós-Graduação em Filosofia da UFT - n. 2 - 2018 
La estructura de este juicio parece clara:

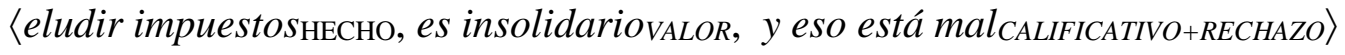

En ese enunciado hemos asignado un valor como fruto de un proceso de identificación y además hemos calificado ese valor como fruto de un proceso de evaluación. Pero es frecuente que el resultado de esos dos procesos no se exprese explícitamente. Se trata de juicios en los que se han producido ciertas supresiones de algunos componentes en esas asignaciones. Son juicios sincopados. Para apreciarlo se requiere que distingamos entre estructura superficial y estructura profunda de los juicios de valor.

\section{Escamoteo de la identificación y ahorro de la evaluación}

Las supresiones que dan lugar a los juicios sincopados se aprecian en la estructura superficial del juicio de valor bajo la forma de ausencia de algún componente que sí se encontraba en la estructura profunda del mismo juicio. No obstante, el usurario y el teórico pueden detectar su presencia a través de un rastro que el componente suprimido ha dejado en la estructura superficial. El análisis formal de esos juicios requiere que le asignemos una estructura profunda en la que aparezca el componente que se suprime en el tránsito hasta la estructura superficial y también que le asignemos una estructura superficial en la que aparezca una huella de ese componente suprimido. Como supresiones frecuentes tenemos el escamoteo de la identificación del valor y el ahorro de la evaluación del valor. ${ }^{2}$

En el escamoteo de la identificación del valor se asigna el calificativo resultado de la evaluación y correspondientes al valor a un hecho en lugar de al valor. El siguiente es un ejemplo de este tipo de juicios:

eludir impuestos está mal

Su estructura superficial aparente sería:

\footnotetext{
${ }^{2}$ Estas nociones de estructura profunda y estructura superficial tienen aquí un sentido análogo al que encontramos en el campo de la lingüística teórica, en particular en la corriente generativa transformacional desarrollada por iniciativa de Noam Chomsky (CHOMSKY, 1955 y 1965, PERIS-VIÑÉ, 2011 y 2012).

Perspectivas - Revista do Programa de Pós-Graduação em Filosofia da UFT - n. 2 - 2018
} 
En estos juicios se califica un hecho (por ejemplo, con un calificativo con carga moral) aunque en realidad el calificativo va dirigido a cierto valor que se realiza en ese hecho. No nombramos el valor, no aparece en la estructura superficial del juicio, pero debemos asumir que en esa estructura superficial hay una traza, una huella de algún valor realizado en ese hecho. Es decir, la estructura profunda y la estructura superficial de esos juicios sincopados son más complejas de lo que podemos suponer. Su estructura profunda es:

\section{$\left\langle\right.$ eludir impuestos $\mathrm{HECHO}, X_{\mathrm{VALOR}}$, está mal CALIFICATIVO+RECHAZO $\rangle$}

Mientras que su estructura superficial real es:

$\langle\text { eludir impuestos } \mathrm{HECHO} \text {, [huella del valor } X]_{\mathrm{VALOR}}$, está mal CALIFICATIVO+RECHAZO $_{\text {i }}$

Este fenómeno del escamoteo de la identificación del valor puede tener la consecuencia perniciosa de hacernos creer que, en estos juicios de valor, en los que el valor no aparece, el calificativo que aparentemente asignamos al hecho es el valor en cuestión. Y, generalizando, creer, erróneamente, que, dado que asignamos calificativos evaluativos sin mencionar valores, los valores tienen una constitución evaluativa, y consiguientemente que están constituidos por el bien o por el mal. Pero en realidad el bien y el mal (y conceptos evaluativos semejantes) no son valores. En los casos de escamoteo del valor, son la resolución final de procesos de identificación de valores y evaluación de valores en cuya formulación lingüística por parte de los usuarios se ha suprimido parte del resultado de esos procesos, la referencia al valor. La supresión de esos resultados en los juicios de valor no implica que tales resultados no formen parte de la estructura de los actos valorativos. Una manera de evitar este error es distinguir entre la estructura superficial y la estructura profunda de los juicios de valor y detectar así, a través de su huella, el valor suprimido en estos casos en la estructura superficial pero que opera en el nivel de la estructura profunda.

En el ahorro de la evaluación del valor se asigna un valor a un hecho y no se explicita el calificativo que nos merece el valor, quizás porque se asume que en el 
significado compartido de ese valor existe ya una carga evaluativa que lo constituye. El siguiente es un ejemplo de este tipo de juicios:

\section{eludir impuestos es insolidario}

Su estructura profunda es:

〈eludir impuestos ${ }_{H E C H O}$, es insolidario ${ }_{V A L O R}$, evaluación implícita de insolidario CALIFICATIVO

Y su estructura superficial aparente sería:

\section{$\langle$ eludir impuestos $H E C H O$, es insolidarioVALOR $\rangle$}

En estos casos nos ahorramos hacer explícita nuestra evaluación. Pero estrictamente, la estructura superficial real debe contener una huella de esa evaluación asumida en la estructura profunda. De lo contrario no podríamos explicar por qué en ocasiones rechazamos la insolidaridad que supone eludir impuestos (por ejemplo en regímenes políticos justos) y en otras ocasiones aceptamos esa insolidaridad que conlleva eludir impuestos (por ejemplo en regímenes políticos injustos y tiranos). Es decir, en la estructura superficial de esos juicios debe aparecer la huella de la evaluación implícita presente en la estructura profunda. Su estructura superficial real es:

\section{〈eludir impuestos ${ }_{H E C H O}$, es insolidario VALOR,}

[huella de la evaluación implícita de insolidario] CALIFICATIVO

Este fenómeno del ahorro de la evaluación puede tener la consecuencia perniciosa de hacer creer, erróneamente, que, dado que asignamos valores con una carga evaluativa implícita, los valores tienen una constitución evaluativa, y consiguientemente que están constituidos por el bien o por el mal. Pero, insistimos, el bien y el mal (y conceptos evaluativos semejantes) no son valores. En los casos de ahorro de la evaluación del valor, son la resolución final de procesos de identificación de valores y evaluación de valores en cuya 
formulación lingüística por parte de los usuarios se ha suprimido parte del resultado de esos procesos, a saber, la expresión explícita de nuestra aceptación o nuestro rechazo del valor. La supresión de esos resultados en los juicios de valor no implica que tales resultados no formen parte de la estructura de los actos valorativos. De nuevo, una manera de evitar este error es distinguir entre la estructura superficial y la estructura profunda de los juicios de valor y apreciar así la evaluación implícita que en estos casos opera en el nivel de la estructura profunda y que nos hemos ahorrado de exponerla en la estructura superficial.

\section{Los valores como propiedades teóricas de los hechos}

La pregunta sobre qué son los valores se responde frecuentemente en la literatura especializada diciendo que son propiedades de cierto tipo. Las diferencias entre las distintas posiciones comienzan cuando hay que decir qué tipo de propiedades son los valores. ${ }^{3}$

Los filósofos suelen identificar tres reinos en el mundo: el físico, el mental y el ideal. Y han caracterizado propiedades propias de cada uno de ellos: propiedades físicas, propiedades psicológicas y propiedades esenciales. La historia de la filosofía podría trazarse como una sucesión de intentos por fundamentar el ámbito de la realidad, el ámbito del conocimiento y el ámbito de la acción a partir de esos tres tipos de propiedades. Diferentes corrientes de pensamiento han priorizado alguna de esas propiedades para fundar el resto. Muestra de ello son las distintas posiciones nominalistas, conceptualistas y realistas en la historia del pensamiento. Esas corrientes se han manifestado igualmente en las teorías axiológicas. Y esto se evidencia en que encontramos diversos intentos de reducir los valores a propiedades físicas, a propiedades psicológicas o a propiedades esenciales. Las discusiones sobre las posibilidades de estos proyectos de reducción forman parte de los acercamientos clásicos al estudio de los valores. En los acercamientos más recientes encontramos intentos de comprender lo peculiar de los valores manteniendo que se trata de propiedades de las cosas, pero evitando reducirlos a algunos de esos tres tipos clásicos de propiedades, aunque intentando expresar cómo se vinculan con cada una de ellas. Por mi

\footnotetext{
${ }^{3}$ En la antología de textos de Shafer-Landau y Cuneo (2007) se recogen las principales propuestas sobre el tipo de propiedades que pueden ser los valores morales.

Perspectivas - Revista do Programa de Pós-Graduação em Filosofia da UFT - n. 2 - 2018
} 
parte, dentro de esos acercamientos recientes, lo que defenderé aquí es que estos objetivos los alcanzamos si reconstruimos los valores como propiedades teóricas.

Los filósofos de la ciencia llaman teóricas a ciertas propiedades cuya asignación al mundo no puede hacerse sólo con nuestros recursos observacionales (bien porque no se manifiestan como algo observable o bien porque carecemos de las capacidades de observación requeridas) y se necesita entonces partir de la asunción de su existencia por detrás de los observable y la aplicación de un complejo entramado teórico y tecnológico para la interpretación de los resultados de la observación y la extracción de los valores de tales propiedades a partir de trazas o indicadores observacionales. Para poder manejar con eficacia las dependencias entre las propiedades teóricas y el complejo teórico que hace posible su determinación se requiere disponer, en principio, de una formulación precisa de ese entramado teórico. Por eso los filósofos intentan formular al modo axiomático las teorías científicas de cuyos términos teóricos quieren conocer sus peculiaridades metodológicas y semánticas. Los valores podrían ser un tipo de propiedades teóricas con rasgos semejantes (aunque también con algunos rasgos divergentes) a los de las propiedades teóricas que encontramos en las teorías científicas. ${ }^{4}$

La concepción de los valores que los presenta como propiedades teóricas adquiere plausibilidad cuando comprobamos que la identificación de valores, al igual que otras propiedades, presenta los siguientes rasgos:

1. los valores no actúan si no están realizados en objetos o en hechos;

2. los valores no son identificables de un modo directo (en cualquier sentido de 'directo', mediante alguna capacidad humana específica y de acción inmediata, o sin relación mediadora con otras propiedades), sino que su identificación pasa por identificar ciertos efectos o trazas de su existencia;

3. los valores no consisten en sucesos, prácticas, acciones u objetos, sino en algo que adscribimos a éstos, por lo que la mera descripción de hechos y objetos no vale como identificación de un valor;

\footnotetext{
${ }^{4}$ La preocupación por los conceptos teóricos es antigua, pero como problema central para la filosofía de la ciencia se consolida al final del primer tercio del siglo XX (CARNAP, 1928 y 1936-1937). Algunos de los hitos más sobresalientes del desarrollo posterior de la discusión los encontramos en (HANSON, 1958), que insiste en la carga teórica de toda observación, en (PUTNAM, 1962), que rompe con la dicotomía entre observacional y teórico, y en (HEMPEL, 1973), que diluye el problema de la teoricidad al contraponer lo teórico no a lo observacional sino a lo pre-teórico.

Perspectivas - Revista do Programa de Pós-Graduação em Filosofia da UFT - n. 2 - 2018
} 
4. la caracterización de un conjunto de hechos no da lugar a la identificación de un valor;

5. no existe un conjunto, máximo, cerrado de valores, pues el mundo no nos limita la conceptualización ni la adscripción de valores al mundo;

6. la identificación de los valores pasa por identificar ciertas relaciones entre los componentes de los hechos en los que se realizan y también ciertas relaciones con otros valores;

7. la identificación de los valores requiere un determinado y complejo cuerpo ideológico previo que establezca los componentes y las relaciones relevantes.

Estos rasgos de la valores no sólo hacen posible su equiparación con propiedades cognoscibles en general, sino que los asemeja a esas propiedades cuando las reconstruimos como propiedades teóricas. Nótese que la semejanza ontológica estaría soportada en la semejanza epistemológica, es decir, en la semejanza entre los procesos de identificación de los valores y los procesos de identificación de las propiedades teóricas. Por tanto, lo que defiendo, de un modo más específico, es que en los procesos de identificación de ciertas propiedades cognoscible denominadas propiedades teóricas y en los procesos de identificación de los valores se emplean recursos cognitivos parcialmente semejantes y que también son semejantes las dificultades que conllevan. De este modo, la concepción de los valores como propiedades de los hechos y de las acciones adquiere plausibilidad cuando logramos equiparar los valores a propiedades teóricas. Esta equiparación será admisible si logramos usar los recursos empleados en la identificación de las nociones teóricas de las teorías científicas para reconstruir de manera adecuada la identificación de valores.

\section{Red de valores y de sus realizaciones}

Antes de su aparición en el mundo, un valor es una entidad conceptual que puede o no realizarse en hechos. Los valores se realizan en hechos y los hechos realizan valores. Una idea básica en esta concepción de los valores es que la identidad de un valor se constituye desde sus realizaciones. No es suficiente su conceptualización, aunque sabemos que es previa y necesaria, ni es necesario esperar a su evaluación, aunque sabemos que es usual. También es usual y comprensible que los usuarios tengan la intención de realizar ciertos valores en ciertos campos de aplicación posible. Pero la identidad del valor a realizar Perspectivas - Revista do Programa de Pós-Graduação em Filosofia da UFT - n. 2 - 2018 
también es previa e independiente de esta usual pretensión de realizarlo. Los valores y sus realizaciones pertenecen a niveles ontológicos, epistémicos y pragmáticos de un orden diferente. Niveles entre los que ciertamente se dan interdependencias. Cabría caracterizar esas posibles interdependencias en términos generales o desentrañarlas respecto de algún entramado particular de valores. Asumimos pues que los valores y sus realizaciones aparecen en redes complejas, en las que sus peculiaridades ontológicas, epistémicas y pragmáticas se muestran entrelazadas.

La conexión de realización básica $r$ (...realiza...), se da entre hechos y valores. Se trata de una relación diádica que asigna valores a hechos. La definimos así:

$$
r=\{\langle h, v\rangle: \text { el hecho } h \text { realiza el valor } v\}
$$

El gráfico de una red correspondiente a un valor y a su realización sería el siguiente:

$$
\text { valor }
$$

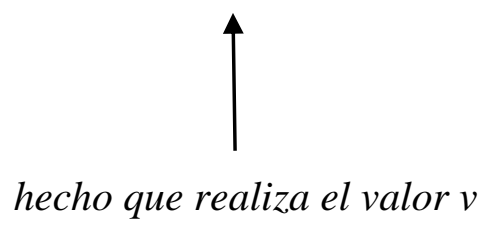

El dominio de la relación $r$ es el conjunto de todos los hechos que realizan algún valor:

$$
\mathrm{D} r=\{h: \exists v\langle h, v\rangle \in r\}
$$

El rango de la relación $r$ es el conjunto de todos los valores que son realizados por algún hecho:

$$
\mathrm{R} r=\{v: \exists h\langle h, v\rangle \in r\}
$$

Perspectivas - Revista do Programa de Pós-Graduação em Filosofia da UFT - n. 2 - 2018 
La inversa de $r$, la relación $r^{-1}$, (...es realizado en...), asignará a cierto valor un hecho en el que se realiza.

$$
r^{-1}=\{\langle v, h\rangle:\langle h, v\rangle \in r\}
$$

La relación $r$, que indica qué valor es realizado por cierto hecho, y la relación $r^{-1}$, que indica qué hechos realizan cierto valor, sirven para representar el resultado de la identificación de valores. Ni $r$ ni $r^{-1}$ son funciones, pues un mismo hecho puede realizar distintos valores y un mismo valor puede realizarse en hechos distintos. Esto refleja el que, en general, no podamos hablar de $e l$ valor que un hecho realiza, ni de $e l$ hecho en el que un valor se realiza. No obstante, sí podemos hablar del conjunto de valores que un hecho realiza y del conjunto de hechos en los que un valor se realiza. Respectivamente:

$$
\begin{gathered}
r[h]=\{v:\langle h, v\rangle \in r\} \\
r^{-1}[v]=\left\{h:\langle v, h\rangle \in r^{-1}\right\}
\end{gathered}
$$

Por ejemplo, los valores cooperación, libertad y realización personal podrían realizarse en diferentes hechos tales como acciones conjuntas de un grupo de individuos y actividades de un individuo.

Aunque venimos insistiendo en que la relación de realización se da entre hechos y valores tenemos que contemplar los casos en los que un valor se realiza no en un hecho sino en otro valor. Por ejemplo, podríamos aceptar que el valor libertad realiza el valor $p a z$, y que a su vez el valor paz realiza el valor felicidad. En forma de red:

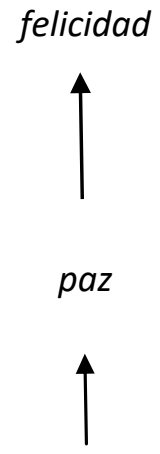

Perspectivas - Revis

a UFT - n. 2 - 2018 
Los valores paz y felicidad serían derivados del valor libertad, éste podría concebirse como un medio para alcanzar aquellos. Por tanto, deberíamos definir una conexión de realización derivada, $r^{\prime}$, entre valores, en la que un valor se realiza en otro valor, no en un hecho. También deberíamos dar cabida a valores colaterales a cierto valor $v$. Sería aquellos que, aunque no realizan a $v$ ni son realizados por $v$, no obstante son realizados por los mismos hechos o valores que realizan a $v$. Aparece así una red compleja de valores y realizaciones, más compleja que la simple conexión básica de partida hecho-valor:

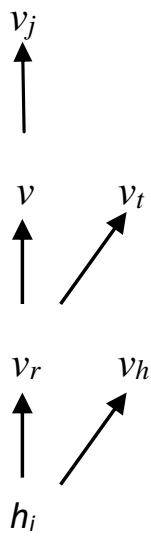

Para un valor $v$ :

- $h_{i}$ representa el conjunto de hechos que realizan al valor $v$;

- $v_{r}$ representa el conjunto de valores que realizan al valor $v$;

- $\quad v_{j}$ es el conjunto de valores realizados por $v$, los valores que se derivan de $v$;

- $\quad v_{h}$ es el conjunto de valores que realizan los hechos que realizan a $v$ pero que ni realizan a $v$ ni son realizados por $v$;

- $\quad v_{t}$ es el conjunto de valores que realizan los valores que realizan a $v$ pero que ni realizan a $v$ ni son realizados por $v$;

- $\left\{v_{h} \cup v_{t}\right\}$ son los valores colaterales a $v$;

- $\left\{\{v\} \cup v_{r} \cup v_{h} \cup v_{t} \cup v_{j}\right\}$ es el conjunto total de valores que los hechos de $h_{i}$ realizan. 


\section{Las ideologías y su papel en la identificación y evaluación de valores}

Un tipo de dificultades que encontramos en el estudio de los valores proceden de las complejas interrelaciones que se dan en el seno de las redes de diversos niveles formadas por valores y sus realizaciones, y que a veces se articulan en ideologías. Pues, aunque cabe usar y analizar los valores uno a uno, lo más propio es usarlos y analizarlos en tanto que integrados en redes jerárquicas. Algunas de esas redes pueden articularse con otros componentes hasta constituir una ideología.

Aunque el concepto de ideología se ha analizado prioritariamente en relación a su papel en ámbitos humanos específicos, tales como la cultura, la economía, la política, la ley, el lenguaje, o la vida cotidiana, nuestros intereses de partida son más generales y no se restringen a un ámbito específico. Aceptamos que una ideología es algo que se manifiesta en un terreno específico de la práctica, pero queremos analizar el concepto de ideología en relación con la práctica en sentido amplio. Disponer de un concepto general de ideología, comprender su naturaleza general, es un requisito para apreciar el papel que las ideologías juegan en ámbitos específicos. También es común en las tradiciones de pensamiento que se vienen ocupando del concepto de ideología preguntarse si las ideologías tienen una influencia negativa, positiva o neutral en la constitución y organización de los distintos ámbitos en que aparecen. Una variante de esta preocupación por el papel de las ideologías es la cuestión de si aportan conocimiento o en cambio distorsionan y producen ilusiones más o menos interesadas. Tampoco estos planteamientos van a ser prioritarios en nuestro acercamiento. Si bien al disponer de un concepto general de ideología sería posible analizar su papel de constitución y de conocimiento.

Vamos a concebir una ideología como una red articulada de valores que incluye una apuesta teórica y una apuesta práctica. La articulación de los valores ser efectúa a veces de modo explícito y metódico, aunque otras veces está sólo bosquejada o implícitamente asumida. Esta noción básica de lo que es una ideología pone el acento inicial en el contenido y en la estructura de las ideologías y no en su origen o en sus funciones. Pero a la vez trata de comprender ciertos usos de las ideologías mostrando la interdependencia existente entre la estructura y el uso de las ideologías. Esa interdependencia se basa a la postre en la naturaleza teórico-práctica de las ideologías. Básicamente, en la apuesta teórica se formulan ciertos principios axiológicos y se identifican los hechos del mundo 
que pueden realizar tales principios, mientras que en la apuesta práctica ciertos agentes optan por la implementación efectiva de algunos de esos principios.

\subsection{Apuesta teórica e identificación de valores}

La apuesta teórica de una ideología contiene dos partes: una vinculación entre la realización de determinados valores y una pretensión de que esa vinculación se aplica o tiene lugar en cierto ámbito de la realidad.

La primera parte es un núcleo conceptual y formal que establece interdependencias entre la realización de los valores de los que se ocupa la ideología. El contenido concreto de esas interdependencias varía de una ideología a otra incluso cuando afecta a los mismos valores. Tales interdependencias se expresan mediante ciertos enunciados generales que a modo de leyes o principios establecen las conexiones entre los varios valores de los que se ocupa la ideología y sus realizaciones. Esas interdependencias se formulan en términos de la noción de realización y de ciertas operaciones sin contenido axiológico, tales como la suma de valores (la libertad más la autonomía), la supresión de valores (la libertad menos la cooperación) o la coexistencia de valores (la libertad junto a la alimentación). Algunos tipos de principios que aparecen en las ideologías son los siguientes:

tipo 1: el valor $v_{1}$ realiza el valor $v_{2}$

tipo 2: la suma de los valores $v_{1}, \ldots, v_{n}$ realiza el valor $v_{x}$

tipo 3: la supresión del valor $v_{z}$ de los valores $v_{1}, \ldots, v_{n}$ realiza el valor $v_{x}$

tipo 4: la coexistencia de los valores $v_{1}, \ldots, v_{n}$ realiza el valor $v_{x}$

La pretensión de aplicar una ideología, la segunda parte de una ideología consiste en afirmar que ciertos sistemas del mundo realizan la vinculación entre valores expresada en los principios de esa ideología. Se trata de un compromiso respecto de en qué parcelas del mundo podría realizarse esa ideología y en cuáles no.

En la apuesta teórica de una ideología va implícita una guía de identificación de valores. Esta identificación ideológica de valores se produce implícitamente respecto de aquellos valores que satisfacen los principios de la ideología en cuestión, concretamente al llevar a cabo las operaciones no axiológicas en términos de las cuales se formulan los principios de una ideología. Realizadas esas operaciones sobre un sistema determinado del mundo y comprobado que el resultado satisface los principios axiológicos, podremos decir Perspectivas - Revista do Programa de Pós-Graduação em Filosofia da UFT - n. 2 - 2018 
que los valores con los que se satisface los principios son los que pretendíamos identificar. Se trata de un caso de la identificación implícita de conceptos mediante el hallazgo de un modelo del constructo teórico al que pertenece el concepto. Los modelos de una ideología serán sistemas del mundo en los que se realizan las dependencias entre los valores y sus realizaciones con las que esa ideología se compromete y que son expresadas en sus principios.

\subsection{Apuesta práctica y evaluación de valores}

La apuesta práctica de una ideología tiene lugar en torno a varios componentes: una red de valores y realizaciones elegidos como meta, un agente que elige, un compromiso de acción y una estrategia de implementación efectiva (a veces con instrumentos coercitivos de diverso tipo) de la vinculación entre valores expresada en sus principios o de la vinculación entre estos principios y las opciones del agente. Para que haya una apuesta práctica se requiere que la ideología incorpore al menos una elección axiológica, es decir unos valores y realizaciones meta. El resto de los componentes prácticos pueden estar presentes o no, y en distinta medida. Además, es importante reconocer que existen ideologías que no conllevan una apuesta práctica. Son instrumentos de conocimiento de valores y no de evaluación de valores y puesta en práctica de los mismos.

Los valores y realizaciones meta elegidos en una ideología son los que condicionan y guían la evaluación posterior de otros valores y realizaciones en atención a las relaciones que mantengan con ellos. Básicamente esa evaluación consiste en elegir los valores y realizaciones que conduzcan a obtener los valores y realizaciones meta elegidos en la apuesta práctica. Para comprender esos procesos de evaluación tengamos en cuenta la red de valores y realizaciones usada para dar cuenta de los procesos de identificación de valores.

La evaluación de un valor conlleva al menos tres fases: una de contrastación de valores, una fase de comparación de valores y una fase de elección de valores. La contrastación de un valor $v$ situado en cierto nivel $n$ de una red de valores consiste en identificar algunos de los siguientes componentes: las realizaciones de $v$ en los niveles inferiores $n-i$, los valores realizados por $v$ en niveles superiores $n+j$, y los valores colaterales a $v$ tanto en su mismo nivel como en niveles inferiores y en niveles superiores. La elección, como resultado de todo el proceso de evaluación, se produce como Perspectivas - Revista do Programa de Pós-Graduação em Filosofia da UFT - n. 2 - 2018 
consecuencia de los resultados de las fases anteriores. Un caso básico sería aquel en el que queremos optar entre dos valores $v_{1}$ y $v_{2}$. La elección de uno de los dos valores $v_{1} \mathrm{y} v_{2}$ tendrá lugar tras la comparación de los resultados de la contrastación de cada uno de ellos, y en atención a la correspondencia de esos resultados con los valores y realizaciones meta. Por ejemplo, un agente elegirá $v_{1}$ en lugar de $v_{2}$ debido a que tras su contrastación y comparación llegue a alguna de estas conclusiones: son preferibles las realizaciones de $v_{1}$ a las de $v_{2}$, por ser correspondientes a las realizaciones meta de su ideología; son preferibles los valores realizados por $v_{1}$ a los de $v_{2}$, por ser correspondientes a los valores meta de su ideología; son preferibles los valores colaterales de $v_{1}$ a los de $v_{2}$, por ser correspondientes a los valores meta de su ideología.

Es necesario tener en cuenta que en los procesos de elección de valores las decisiones se adoptan a veces en atención a la preferencia de valores meta relativos y a veces en atención a la preferencia de valores meta absolutos. El segundo caso es el que a veces es denominado por opciones puras.

Una ideología conlleva una guía de evaluación de valores al incluir valores y realizaciones meta en su apuesta práctica con los que contrastar los valores sometidos a evaluación. Se trata por ello de una evaluación ideológica.

\subsection{Independencia e interacción entre la apuesta teórica y la apuesta práctica de una ideología}

La apuesta práctica y la apuesta teórica de una ideología pueden ser independientes o pueden interactuar de distintas maneras. La independencia se manifiesta en la existencia de enunciados teóricos, que expresan conexiones no evaluativas entre valores, y de enunciados evaluativos, que expresan opciones y evaluaciones de algún agente. Ejemplos de enunciados teóricos serían los siguientes: el valor $v_{1}$ realiza el valor $v_{2}$; la supresión del valor $v_{z}$ de los valores $v_{1}, \ldots, v_{n}$ realiza el valor $v_{x}$; la coexistencia de los valores $v_{1}, \ldots, v_{n}$ realiza el valor $v_{x}$. Ejemplos de enunciados evaluativos serían los siguientes: el valor $v_{1}$ es preferible al valor $v_{2}$; el valor $v$ es bueno; las realizaciones $v_{i}$ del valor $v_{1}$ son preferible a las realizaciones $v_{h}$ del valor $v_{2}$.

La interacción aparece cuando la evaluación de valores (resultado central de la apuesta práctica) se ve afectada por la identificación de valores (resultado central de la apuesta teórica) en la medida en que cierto agente use los principios teóricos de una Perspectivas - Revista do Programa de Pós-Graduação em Filosofia da UFT - n. 2 - 2018 
ideología (o su satisfacción en ciertos modelos) para guiar sus elecciones. Pero, en cualquier caso, estas conexiones entre identificación y evaluación, entre la apuesta teórica y la apuesta práctica de una ideología, entre sus principios teóricos y las opciones de los agentes, serán conexiones a los ojos de un agente, no se siguen exclusivamente a partir de los principios teóricos de una ideología. Esta interacción se expresa en enunciados mixtos, los cuales conectan las opciones y las evaluaciones de un agente con el contenido de algún principio teórico. Un ejemplo de enunciado mixto sería el siguiente: el valor $v_{l}$ es preferible al valor $v_{2}$ porque: $v_{1}$ realiza $v_{3}, v_{2}$ no realiza $v_{3}$ y realizar $v_{3}$ es preferible a no realizarlo.

\subsection{La dependencia ideológica de la identificación y de la evaluación de valores}

Tanto los procesos de identificación de valores como los procesos de evaluación de valores son ideológico-dependientes, en el sentido de que tales procesos están guiados y están condicionados por una ideología. Pero existe una gran diferencia. La identificación de un determinado valor está guiada por la apuesta teórica de una ideología, no por su apuesta práctica. Y por tanto los procesos de identificación de valores son ideológico-dependientes, pero no son evaluativo-dependientes. Es decir, la identificación de valores requiere partir de una ideología, pero no requiere partir de los compromisos axiológicos de su apuesta práctica. Por su parte, la evaluación de un determinado valor sí está guiada por la apuesta práctica de una ideología, es decir, está guiada por los compromisos axiológicos de esa apuesta práctica.

\section{Síntesis y principios de la concepción semántica de los valores}

Hasta aquí el esbozo de la concepción general sobre los valores defendida en este texto. Esta concepción puede verse como el desarrollo de ciertos principios. Algunos de estos principios pueden resultar fáciles de aceptar o cuasi evidentes. Su relevancia se manifiesta al ser aplicados de manera encadenada. Los principios encadenados de esta concepción, de ser adecuados, implican consecuencias relevantes para la comprensión de la naturaleza de los valores. A continuación, los formulamos sintéticamente.

Principio de la factualidad de la realización de los valores: los valores se realizan en hechos.

Perspectivas - Revista do Programa de Pós-Graduação em Filosofia da UFT - n. 2 - 2018 
Principio de la independencia intencional de la realización de valores: la realización de los valores en los hechos se produce independientemente del compromiso de los agentes con los valores fruto de su evaluación y expresado en un juicio de valor.

Principio de la independencia evaluativa de la identificación de valores: la identificación de valores en los hechos es posible efectuarla sin necesidad de evaluar esos valores.

Principio de la identificación cognitiva de los valores: la identificación de la realización de los valores independiente de la evaluación es posible con el empleo de los recursos cognitivos usuales para conocer propiedades del mundo.

Principio de la dependencia ideológica de la identificación de valores: la posibilidad de la identificación cognitiva de los valores requiere necesariamente el empleo instrumental de cierta ideología; derivadamente, ciertos valores no podrán ser identificados, resultarán invisibles, si no se parte de la ideología correspondiente.

\section{Los valores desde la metateoría estructural}

¿Cómo fundamentar el papel que juegan las ideologías en los procesos de identificación y de evaluación de valores que acabamos de analizar? Abordaré un aspecto particular de esa cuestión general, el que afecta al principio de la dependencia ideológica de la identificación de valores. Mi propósito es usar algunos recursos de la metateoría estructural para fundamentar ese principio. ${ }^{5}$

La metateoría estructural, el principal y más desarrollado de los enfoques semánticos de la ciencia, prioriza las propiedades semánticas de las piezas de conocimiento científico, en particular de las teorías, y lo hace recurriendo a la teoría de modelos. Las propiedades

\footnotetext{
${ }^{5}$ La metateoría estructural viene desarrollándose con gran profusión desde su obra inicial (SNEED, 1971) en varias direcciones. (BALZER; MOULINES \& SNEED, 1987) sigue siendo la referencia, ya clásica, de una formulación precisa y amplia de los recursos y posibilidades propios de la metateoría estructural. En algunas ocasiones se han publicado listas de las publicaciones que siguen esta corriente. La última de esas listas bibliográficas ha sido (ABREU; LORENZANO \& MOULINES, 2013). Los autores en lengua castellana han tenido desde su inicio una presencia considerable en la metateoría estructural. Muestras de ello se encuentra en las recopilaciones de artículos de Díez y Lorenzano (2002) y Peris-Viñé (2012).

Perspectivas - Revista do Programa de Pós-Graduação em Filosofia da UFT - n. 2 - 2018
} 
semánticas de un constructo cognitivo (una teoría o un concepto) se manifiestan en sus realizaciones, las cuales son caracterizadas como modelos en el sentido de la teoría de modelos. La identidad de un constructo se constituye pues desde sus modelos. Nuestra concepción de valores como propiedades teóricas, al priorizar los procesos de identificación, puede beneficiarse de esta capacidad de los enfoques semánticos de desentrañar la identidad de un concepto o de una teoría a partir de sus realizaciones. Por tanto, si logramos reconstruir esta concepción de los valores mediante la metateoría estructural ello será un apoyo para la idea de que la identidad de un valor se constituye desde sus realizaciones.

La relación modelística (semántica) fundamental en las ciencias empíricas viene expresada por la aserción empírica asociada a toda teoría empírica. Básicamente es una afirmación de que ciertos sistemas del mundo satisfacen la teoría. Es decir, expresa la conexión semántica entre la teoría y su significado, representado éste por los sistemas en los que aquélla puede mostrarse verdadera, sistemas a los que se denominan modelos. Así, tenemos una teoría, sus modelos y una aserción semántica que expresa un compromiso de que ciertos sistemas del mundo son modelos de la teoría. Las teorías se realizan en sus modelos, y mediante este tipo de aserciones semánticas de carácter empírico expresamos nuestros compromisos al respecto.

Desde un enfoque semántico, ni las teorías ni sus modelos son verdaderos o falsos. Son objetos complejos que podemos usar como instrumentos para expresar ciertas relaciones de naturaleza cognitiva con el mundo. Ni los objetos ni los instrumentos son cosas que puedan ser verdaderas o falsas. Lo que puede ser verdadero o falso son las aserciones semánticas. Respecto de los valores cabría afirmar lo mismo: ni los valores, ni las ideologías en las que se integran, ni sus realizaciones, son verdaderos o falsos. Lo que puede ser verdadero o falso es cierto enunciado que asevere que cierto hecho (objeto, acto o suceso) realiza determinado valor, o realiza determinada conexión entre ciertos valores, expresada en un enunciado que los relacione o en una agrupación reticular de tales enunciados que forme determinada ideología. Es decir, lo verdadero o falso es una aserción semántica que asevere que cierta situación realiza cierto valor individual aislado o que cierta situación compleja es un modelo de cierto enunciado relacional o de cierta ideología. Los modelos de una ideología serán sistemas del mundo en los que se realizan las 
dependencias entre los valores y sus realizaciones con las que ese enunciado o esa ideología se comprometen.

\section{Lo observacional, lo teórico, lo neutral y lo ideológico}

Son conocidos los inconvenientes que en la teoría de la ciencia ha ocasionado la concepción de la observación como acceso directo conceptualmente no mediado a los hechos, y la consiguiente propuesta de una dicotomía entre observacional y teórico. La siguiente es una lista informal de tales inconvenientes: asumir que tiene sentido plantearse el desiderátum de un acceso observacional directo a los hechos; considerar que es posible un acceso observacional directo a los hechos; no apreciar la carga teórica de la observación; asumir que, dada la carga teórica de la observación, ante los mismos hechos es posible producir cualesquiera informes de observación, incluso contradictorios aunque igualmente adecuados; concebir los conceptos observacionales exclusivamente como conceptos no teóricos; concebir los conceptos no-teóricos exclusivamente como conceptos observacionales; incomprensión de los procesos de cambio que implican la transformación de los conceptos teóricos en conceptos no-teóricos

Con el tiempo y la aportaciones de filósofos de la ciencia de distintas corrientes (historicistas y semánticas, principalmente) esa dicotomía se ha reconvertido en las distinciones observacional // no-observacional y teórico // no-teórico. Es decir, lo opuesto a lo observacional no es lo teórico, por lo que pueden existir conceptos observacionales y a la vez teóricos. Ni lo no observacional es necesariamente teórico, por lo que pueden existir conceptos no observacionales y a la vez no teóricos. A la base de estos cambios ha estado una concepción de la teoricidad de los conceptos científicos como una cualidad relativa a la teoría en la que se emplean los conceptos afectados. El desarrollo de esa nueva concepción de la teoricidad es uno de los resultados más innovadores y relevantes obtenidos en las discusiones sobre la relación entre observación y teoría que han tenido lugar desde las tres últimas décadas del siglo XX. ${ }^{6}$

\footnotetext{
${ }^{6} \mathrm{El}$ análisis de la teoricidad estuvo en el inicio de la metateoría estructural, y es posiblemente el terreno donde las aportaciones de esta corriente son más sólidas y relevantes. Las propuestas originales y que marcaron el camino de las sucesivas propuestas se encuentran en (SNEED, 1971). Una versión mejorada aparece en (STEGMÜLLER, 1973). Pero es en (BALZER \& MOULINES, 1980) donde se hacen las modificaciones de mayor calado, y se logra afianzar el aparato modelo teórico en el tratamiento de nociones centrales para la formulación de un criterio de teoricidad que aspiraba a trascender una formulación meramente Perspectivas - Revista do Programa de Pós-Graduação em Filosofia da UFT - n. 2 - 2018
} 
La justificación de esta concepción de la teoricidad procede del análisis de los procedimientos de determinación de los valores de los conceptos afectados. Este es uno de los terrenos en el que la metateoría estructural ha hecho las contribuciones más decisivas. La metateoría estructural nos ha enseñado que la determinación de la magnitud de ciertas nociones presupone asumir la validez de cierta teoría $T$. Esto implica que todo procedimiento empleado en determinar la magnitud de tales nociones es un modelo de $T$, es decir, que no puede ser descrito como un sistema del mundo que no satisfaga las leyes fundamentales de $T$. Esas nociones son las $T$-teóricas. Existen otras nociones en $T$ que son no-T-teóricas, en el sentido de que alguno de los procedimientos de determinación de sus magnitudes puede ser descrito como un sistema que no satisface las leyes de $T$. Estas diferencias se expresan mediante un criterio de T-teoricidad. Además, siguiendo el criterio de $T$-teoricidad, cabe la posibilidad de que lo no- $T$-teórico sea a la vez $T$ '-teórico, donde $T^{\prime}$ es distinta y quizás antecedente a $T$ en un sentido temporal o conceptual.

Pues bien, en atención a razones semejantes, propongo que la dicotomía neutral // ideológico se reconvierta en las distinciones neutral // no-neutral e ideológico // noideológico. En metaética encontramos con frecuencia una imagen de lo moral como algo a lo podríamos acceder de un modo neutral, directo y sin mediación conceptual o ideológica. Se asume que ese tipo de acceso es posible y se debate sobre cómo lograrlo. Ese debate se suele apoyar en la defensa de una dicotomía entre neutral e ideológico. Las cuestiones que surgen en esos debates en metaética son comparables con las cuestiones que se han planteado en filosofía de la ciencia sobre el posible acceso observacional directo y conceptualmente no mediado a los hechos, y el consiguiente fundamento para la dicotomía entre observacional y teórico.

Prueba de que las cuestiones en ambos campos son comparables es que los inconvenientes que en la metateoría de la axiología (y en especial en metaética) ha ocasionado el oponer neutral a ideológico son equiparables a los que ha ocasionado en la metateoría de la ciencia el oponer observacional a teórico. La siguiente es una lista informal de tales inconvenientes: asumir que tiene sentido plantearse el desiderátum de un acceso neutral y directo a los valores morales; considerar que es posible un acceso neutral y directo a los valores morales; no apreciar la necesidad de un carga ideológica presupuesta

intuitiva. Los autores que trabajan en esta corriente han seguido haciendo propuestas que buscan ampliar el alcance y facilitar la aplicabilidad del criterio de teoricidad.

Perspectivas - Revista do Programa de Pós-Graduação em Filosofia da UFT - n. 2 - 2018 
en la identificación de valores morales; asumir que, dada la carga ideológica de la identificación moral, ante los mismos hechos es posible producir cualesquiera juicios de valor, incluso contradictorios aunque igualmente adecuados; concebir los conceptos neutrales exclusivamente como conceptos no ideológicos; concebir los conceptos no ideológicos exclusivamente como conceptos neutrales; incomprensión de los procesos de cambio que implican la transformación de los conceptos ideológicos en conceptos no ideológicos.

En ambos terrenos, en el de la ciencia y en el de la moral, está implicado el problema de nuestro acceso objetivo, a los hechos en el caso de la ciencia y a los valores en el caso de la moral. En ambos terrenos está en discusión si ese acceso objetivo podría y debería estar basado en un acceso directo. En el caso de la filosofía de la ciencia las alternativas al acceso observacional directo apelan a la carga teórica de la observación de los hechos. En el caso de la metaética las alternativas al acceso neutral directo deberían apelar a lo podríamos llamar carga ideológica de la identificación de los valores morales. En ambos terrenos el problema es el acceso. Del mismo modo que la consideración de que es posible un acceso observacional a los hechos, sin carga teórica, impide entender los procesos realmente implicados en la determinación de las magnitudes de los conceptos científicos, la consideración de que es posible un acceso neutral a los valores, sin carga ideológica, impide entender los procesos realmente implicados en la identificación de los valores y la posterior defensa de los mismos.

Es decir, si el desarrollo de la nueva concepción de la teoricidad descansa en el análisis y comprensión de los procedimientos de determinación de las magnitudes de los conceptos científicos, la fundamentación de la alternativa a la asunción de un acceso neutral a los valores debe descansar en el análisis y comprensión de los procedimientos de identificación de valores. Insistimos en que la identificación de valores requiere de hechos, pero igualmente requiere de una teoría o ideología que conozca tales hechos. No podemos identificar valores sin hechos y sin ideología, por las mismas razones que la ciencia no puede identificar propiedades en el mundo sin hechos y sin teoría. La identificación de un valor requiere la asunción de ciertos compromisos previos, compromisos que podrán ser más o menos elaborados, más o menos conscientes y explícitos. En ocasiones tales compromisos constituyen una completa ideología, en cuyos principios teóricos se integrará ese valor y otros relacionados con él.

Perspectivas - Revista do Programa de Pós-Graduação em Filosofia da UFT - n. 2 - 2018 


\section{Criterio de ideologicidad}

Además de este enfoque general, según el cual la identificación de valores requiere de alguna ideología, lo que defiendo, como algo más contundente, es que ciertos valores sólo serán identificables desde cierta posición ideológica determinada, sin cuyo concurso no será posible establecer ni tan siquiera su presencia. Son valores dependientes, respecto de su identificación, de esa ideología. No es que su conceptualización, propuesta o defensa requiera partir de una ideología, sino que es su misma identificación lo que está en juego y lo que depende de esa ideología. Estas consideraciones hacen posible en principio trasladar al estudio de los valores los recursos que la metateoría estructural ha desarrollado para distinguir entre nociones $T$-teóricas y no- $T$-teóricas respecto de cierta teoría $T$. En el caso de los valores el resultado esperado sería poder distinguir entre valores I-ideológicos y noI-ideológicos respecto de cierta ideología $I$.

Formulemos un criterio de ideologicidad en línea con el criterio de teoricidad de la metateoría estructural. Recordemos que el criterio de teoricidad para las nociones de una teoría $T$ se formula en términos de la noción de método de determinación para las nociones de $T$, cuya reconstrucción se lograba al mostrarlo como un modelo potencial de $T$. En consonancia con ello el criterio de ideologicidad para los valores de una ideología $I$ se formula en términos de la noción de método de identificación para los valores de $I$, noción cuya reconstrucción se logrará al mostrarlo como un modelo potencial de $I$. El criterio es el siguiente: si todo método de identificación de cierto valor $v$ presupone cierta ideología $I$, diremos que $v$ es I-ideológico; en cambio, si existe un método de identificación de $v$ que no presupone la ideología $I$, diremos entonces que $v$ es no-I-ideológico.

La adecuación de un criterio ideologicidad para el estudio de los valores requiere disponer de las nociones de ideología y de método de identificación de valores. Ambas nociones se han caracterizado más arriba al presentar la concepción general sobre los valores. Básicamente, una ideología es una red de valores respecto de los cuales se formulan ciertos principios axiológicos y se identifican los hechos del mundo que los realizan. Además, las ideologías suelen incorporar una apuesta práctica en la que se opta por ciertos valores y realizaciones a modo de metas. Los métodos de identificación de valores se especifican en términos de las conexiones de realización entre hechos y valores. En la posibilidad de concebir los valores en términos de un criterio de ideologicidad, en Perspectivas - Revista do Programa de Pós-Graduação em Filosofia da UFT - n. 2 - 2018 
línea con el criterio de teoricidad de la metateoría estructural, se expresan dos de los rasgos sustanciales de los valores que la concepción defendida aquí pone de manifiesto: su vinculación con los hechos y su dependencia de las ideologías. Aunque sin duda existen otros rasgos o fenómenos característicos de los valores que podrán ser entendidos a partir de su reconstrucción mediante la metateoría estructural.

\section{REFERENCIAS}

ABREU, C.; LORENZANO, P. \& C. U. MOULINES. Bibliography of Structuralism III (1995-2012 and Additions). Metatheoria, v. 3, n. 2, p. 1-35, 2013.

BALZER, W. \& C. U. MOULINES. On Theoricity. Synthese, v. 44, p. 467-494, 1980.

BALZER, W.; MOULINES, C. U. \& J. D. SNEED. An architectonic for science. The structuralist program. Dordrecht: Reidel, 1987.

CHOMSKY, N. The Logical Structure of Linguistic Theory. New York: Plenum, 1975, [1955].

CHOMSKY, N. Aspect of the Theory of Syntax. Cambridge, Mass.: The MIT Press, 1965.

DÍEZ, J. A. \& P. LORENZANO (eds.). Desarrollos actuales de la metateoría estructuralista: problemas $\mathbf{y}$ discusiones. Bernal: Universidad Rovira i Virgili/Universidad Autónoma de Zacatecas/Universidad Nacional de Quilmes, 2002.

HANSON, N. R. Patterns of Discovery. Cambridge: Cambridge University Press, 1958.

HARE, R.M. The Language of Morals. Oxford: Clarendon Press, 1952.

HEMPEL, C. G. The Meaning of Theoretical Terms: A Critique of the Standard Empiricist Construal. En SUPPES, P. at al. (eds.). Logic, Methodology and Philosophy of Science. IV. Amsterdam: Nort-Holland, 1973, pp. 367-378.

MACKIE, J.L. Ethics: Inventing Right and Wrong. Harmondsworth: Penguin, 1977.

MOORE, G. E. Principia Ethica. Cambridge: Cambridge University Press, 1903.

PERIS-VIÑÉ, L.M. Actual Models of the Chomsky Grammar. Metatheoria, v.1, n. 2, p. 195-225, 2011.

PERIS-VIÑÉ, L. M. Estructura parcial de la gramática estándar del castellano. En PERISVIÑÉ, L.M. (ed.). Filosofía de la ciencia en Iberoamérica: metateoría estructural. Madrid: Editorial Tecnos, 2012, pp. 225-258. 
PERIS-VIÑE, L. M. (ed.). Filosofía de la ciencia en iberoamérica: metateoría estructural. Madrid: Editorial Tecnos, 2012.

PUTNAM, H. What Theories are Not?. En NAGEL, E.; SUPPES, P. \& A. TARSKI (eds.). Logic, Methodology and Philosophy of Science. Stanford: Stanford University Press, 1962, pp. 240-251.

PUTNAM, H. The Collapse of the Fact/Value Dichotomy and Other Essays. Cambridge, MA: Harvard University Press, 2002.

SHAFER-LANDAU, R. \& T. CUNEO (eds.). Foundations of Ethics: An Anthology. Oxford: Blackwell, 2007.

SNEED, J.D. The Logical Structure of Mathematical Physics. Dordrecht: Reidel, 1971.

STEGMÜLlER, W. Probleme und Resultate der Wissenschaftstheorie und analytischen Philosophie, Band II/2: Theorienstrukturen und Theorien-Dynamik. Berlin: Springer, 1973.

WILliaMS, B. Ethics and the Limits of Philosophy. Cambridge, MA: Harvard University Press, 1985. 